\title{
Modèle de gestion moderne de projet : entre tradition et renouvellement théorique
}

\author{
Victor Mignenan ${ }^{\mathrm{a}}$, Brahim Meddeb $^{\mathrm{b}}$
}

RÉSUMÉ. Sur les plans épistémologiques, théoriques et méthodologiques, la gestion moderne de projet est portée par des approches universelles et leurs perspectives critiques, mais aussi par des courants de pensée de l'hypermodernité. L'article met en évidence les modèles paradigmatiques et identifie les cadres théoriques au sein desquels les chercheurs pourraient inscrire leurs travaux. D'abord, la traditionnelle approche universelle positiviste considère le projet comme une entité d'origine pratique. Le gestionnaire y est apprécié selon ses compétences instrumentales, qui optimisent le succès de gestion du projet. En revanche, la perspective contingente met de l'avant la synergie et la collaboration synchronisées des équipes, et elle développe des structures souples pour satisfaire les parties prenantes. En conséquence, l’hypothèse selon laquelle la perspective universelle constitue un meilleur prédicteur du succès des projets que la perspective contingente est un mythe. L'analyse montre que les deux perspectives sont complémentaires, et qu'elles ont à la fois des moteurs et des obstacles pour le succès global du projet.

\begin{abstract}
On the epistemological, theoretical and methodological levels, modern project management is carried by universal approaches and their critical perspectives, but also by currents of thought of hypermodernity. This article highlights paradigmatic models and identifies the theoretical frameworks within which researchers could write their work. The traditional positivist universal approach considers the project as an entity of practical origin. The manager is valued for his instrumental skills that optimize management success of the project. On the other hand, the contingent perspective puts forward the synchronized synergy and collaboration of the teams; it develops flexible structures to satisfy stakeholders. As a result, the assumption that the universal approach is a better predictor of project success than the contingent approach is a myth. The analysis shows that the two perspectives are complementary, and that they have drivers and obstacles for the overall success of the project.
\end{abstract}

\section{Introduction}

Toutes les recherches en gestion de projet visent une problématique particulière et doivent être inscrites dans un cadre théorique ayant un socle ontologique, une perception épistémologique et un cadre méthodologique singulier (Gauthier, 2014; Gauthier et Ika, 2012). Bien qu'elle soit parfois peu explicite, la problématique de recherche en gestion de projet porte souvent sur la performance globale des projets.

Par conséquent, lorsqu'on analyse l'évolution de l'approche universelle en gestion de projet (p. ex., PMI, IPMA, PRINCE2, ISO 21500), on est en mesure de comprendre pourquoi ladite approche connait une amélioration continue. Il en est de même pour la perspective contingente de gestion de l'école scandinave. Cependant, on ignore non seulement quels sont les paramètres théoriques de ces approches, mais aussi les façons d'inscrire un sujet de recherche dans un champ théorique de gestion de projet peu stable.

L'article poursuit deux principaux objectifs. Le premier consiste à dresser le portrait du fondement théorique de ces deux approches de la gestion de projet : caractéristiques, compétences, similitudes et divergences. Le second tente de présenter les assises sur lesquelles les recherches en gestion de projet pourraient reposer.

\footnotetext{
a Docteur en management de projet, membre du laboratoire CAISEN, Université du Québec à Chicoutimi

b Professeur titulaire, Ph. D. relations industrielles, directeur du laboration CAISEN, Université du Québec à Chicoutimi
} 


\section{Portrait du fondement théorique en gestion de projet}

Selon quelques travaux (Asquin, Falcoz et Picq, 2005; Asquin, Garel et Picq, 2010), un projet possède cinq caractéristiques : 1) il est un objet unique (tout projet n'est pas la reproduction à l'identique de ce qui existe déjà); 2) il est singulier (chaque projet est différent et donc novateur); 3 ) il est temporaire (chaque projet est limité dans le temps et dans l'espace : il possède un début et une fin, et se déroule dans un lieu et un contexte spécifiques); 4) il est transversal (chaque projet mobilise et implique plusieurs rapports de force); 5) et il a une idéologie progressiste (chaque projet mobilise des ressources au fur et à mesure qu'il avance pour prendre des décisions éclairées). Or, deux grandes clés de lecture permettent d'appréhender le fondement théorique de la gestion de projet, soit la perspective universelle et la perspective contingente.

\subsection{Perspective universelle traditionnelle de la gestion de projet}

La perspective universelle en gestion de projet s'est précisée au lendemain de la Seconde Guerre mondiale, grâce à des organismes professionnels, notamment le Project Management Institute (PMI; Garel, 2011a, 2011b, 2013; Morris, Pinto et Söderlund, 2011; Morrissette, 2013; Turner, 2014). Les précurseurs de cette perspective théorique considèrent le projet sous l'angle des activités de production. Ils assimilent le cycle de vie du projet à un processus constitué d'un ensemble d'activités planifiées et organisées ayant un début et une fin. Appréhendé comme un objet peu social qui s'adapte difficilement à la réalité, le projet se caractérise par une structure organisationnelle et par un socle normatif qui limitent les acteurs dans leurs démarches de prise de décision éclairée. Les courants de pensée qui s'inscrivent dans cette perspective universelle traitent essentiellement de la problématique associée aux activités de production et au processus de projet.

Dans cette perspective, les pratiques de gestion sont structurées autour de l'école d'optimisation d'inspiration strictement taylorienne. Dans une option de production de structure et de processus, la caractéristique principale est l'utilisation de l'approche systémique de la planification et de contrôle pour optimiser les résultats. On assiste à une forte prédominance des cultures technicistes avec la mobilisation des mathématiques (recherche opérationnelle) pour définir et analyser les projets (Cicmil, 2006; Söderlund, 2004; Špundaka, 2014).

Pour leur part, les parties prenantes au projet manifestent leur volonté de rendre le projet prédictible. Ici, une des caractéristiques est la prévalence des secteurs traditionnels des activités de projet, avec une modélisation systémique et un fort penchant d'optimisation de l'équilibre entre la durée, les coûts et la qualité (Špundaka, 2014; Too et Weaver, 2014).

Les postulats théoriques de la perspective universelle de gestion de projet portent pour l'essentiel sur la définition des meilleures techniques et sur l'application pratique des fonctions de planification, de programmation, d'organisation et de contrôle pour gérer le projet selon un principe d'efficience et de décomposition de tâches complexes (Cicmil et Hodgson, 2006).

Toujours selon cette perspective, la gestion de projet s'apparente à un phénomène prescriptible, isolé de son écosystème et des jeux de pouvoir exercés par les différentes parties prenantes impliquées (Slevin, 2002; Too et Weaver, 2014; Turner, 2010). En conséquence, l'organisation, entité responsable de la combinaison des techniques et pratiques pour atteindre les résultats, se conduit comme un agent rationnel ayant des connaissances instrumentales pour générer de la performance (Too et Weaver, 2014; Turner, 2014; Turner, Huemann et Keegan, 2008).

Enfin, les postulats de la gestion de projet, suivant cette clé de lecture universelle, sont ancrés dans un socle ontologique, un fondement épistémologique ainsi qu'un cadre méthodologique et empirique spécifiques qu’il convient de présenter.

\section{Sur le plan ontologique}

Sur le plan ontologique (l'étude de l'être), la perspective universelle considère le projet comme une réalité concrète, pratique, stable et universelle. En conséquence, il est considéré à la fois comme une entité qui demeure ("étant») susceptible d'être programmée au même titre qu'une "machine». Cependant, plusieurs chercheurs (Sage, Dainty et Brookes, 2013, 2014) admettent la rareté des contributions qui soutiennent l'ontologie en gestion de projet. 


\section{Sur le plan épistémologique}

La perspective universelle remet en question la définition du projet comme sujet ou objet, mais également la manière de parvenir à saisir le sujet (Gauthier et Ika, 2012). Plus spécifiquement, elle est préoccupée par la démarcation entre la connaissance scientifique et les autres formes de connaissances. Cependant, de plus en plus, des précurseurs de cette perspective universelle proposent la connaissance comme le fruit d'interactions humaines (Bredillet, 2006; Bredillet, Yatim et Ruiz, 2010).

\section{Sur le plan méthodologique}

La perspective universelle se concentre sur des approches d'optimisation structurées par les recherches opérationnelles et par les théories statistiques de décision fondées sur des cultures hypothético-déductives. Ainsi, sur le plan des méthodes déployées, on assiste au développement des stratégies quantitatives. Celles-ci sont structurées par des hypothèses objectivistes basées sur la pratique permettant d'optimiser les ressources. Les instruments de suivi et de contrôle du processus (p. ex., les diagrammes PERT et de Gantt) sont préconisés (Cicmil et Hodgson, 2006).

\section{Sur le plan empirique}

Les terrains de manœuvre mis de l'avant sont, entre autres, les secteurs d'ingénierie ou des grands travaux de construction mécaniques, routiers, bâtiments et électriques. Aujourd'hui, la gestion de projet s'élargit pour inclure les secteurs de la santé, de l'éducation, du sport, etc. Le gestionnaire de projet, qui a la responsabilité du succès ou de l'échec, est la principale source de données associées au projet. Sa compétence est ainsi mesurée selon sa maitrise des connaissances instrumentales, des normes, des règles et des procédures appliquées au projet. Cependant, les acteurs du projet font peu partie de la modélisation dans la perspective universelle. De même, on note l'insuffisance de la coopération active entre les partenaires du projet et la faible accession à la créativité de l'équipe de projet.

Bref, l'approche universelle de gestion de projet vise à bâtir des régularités, pour ainsi caractériser les phénomènes qui campent spécifiquement le contenu du projet. Pourtant, le projet, objet de l'application des normes et principes, est de nature instable et conduit dans des environnements en perpétuel mouvement. De même, le fait même d'institutionnaliser la gestion de projet a engendré, d'après plusieurs auteurs (Asquin, Garel et Picq, 2009; Boutinet, 2005), la rigidité et le cloisonnement perceptibles dans les différentes phases du cycle de vie, ce qui néglige, par conséquent, la présence des individus. Ensuite, la formalisation du processus de planification par les diagrammes PERT et de Gantt crée un système rigide dans lequel chaque acteur se limite à suivre à la lettre les procédures. Cela rend le dispositif de gestion peu ouvert et favorise dans une faible mesure les initiatives des acteurs. Enfin, l'approche universelle est moins adaptable pour réagir aux aléas et aux urgences de l'écosystème du projet.

\subsection{Glissement de la perspective universelle vers la perspective contingente}

La bureaucratie, l'objectivation, l'instrumentation, la standardisation et la rationalisation de la perspective universelle de gestion de projet présentent d'énormes lacunes quant au succès global d'un projet (Camilleri, 2011; Müller, 2005; Padgett, 2009; PRINCE2, 2005). En revanche, les professionnels et théoriciens admettent que, dans un monde d'hypermodernité et donc d'agilité ${ }^{1}$, les organisations ont besoin de normes souples, de référentiels et de méthodes plus légers ancrés dans la gestion des exigences contextuelles ainsi que de la prise en compte des parties prenantes (Guédon, 2009; Kerzner, 2014; Loufrani-Fedida, 2011; Špundaka, 2014). Des limites qui émergent de l'approche traditionnelle, une piste alternative de la gestion de projet se pointe en creux pour ainsi répondre à un objet (projet) «nomade », c'est-à-dire en perpétuel mouvement. L'approche contingente de l'école scandinave, avec le développement de l'interdisciplinarité, de la multidisciplinarité et de la transdisciplinarité dans la recherche en gestion de projet, permet d'avoir un regard nouveau sur la performance de projet.

\subsection{Assise théorique de la perspective contingente}

Les lacunes de l'approche universelle traditionnelle ont fait émerger, entre autres, l'école scandinave (approche contingente) et l'agilité (Turner, 2014). Dans cette vision critique empruntée de l'école de Francfort ${ }^{2}$, les précurseurs de cette nouvelle perspective (Sage et collab., 2013, 2014; Sauser, Reilly et 
Shenhar, 2009) considèrent le projet comme un système social, un système adaptatif et un objet d'enjeux. De ce fait, sa signification et son succès varient selon la perception des parties prenantes. Chacun des acteurs de projet, par sa culture et son expérience, percevra différemment le succès du projet (Söderlund, 2011; Söderlund et Geraldi, 2012).

De ce qui précède, il convient de souligner que la perspective contingente de la gestion de projet l'appréhende comme une pratique sociale décloisonnée où l'humain agissant est mis au centre de l'action et est donc intégré au sein de la modélisation (Guédon, 2009; Lapayre, 2010). Il s'agit ici d'une vision renouvelée de gestion du projet, déplaçant ainsi le point focal du postulat «étant» de rationalité vers un socle ontologique et épistémologique du «devenant» (Chia, 1995). L'attention est portée sur des micropratiques et des politiques développées au sein du projet qui influencent les différentes parties prenantes. Les énergies sont concentrées sur l'analyse des acteurs-gestionnaires, qui apprennent et négocient en permanence les structures organisationnelles; discutent des enjeux de coconstruction, de l'implication des bénéficiaires et des jeux de pouvoir qui découlent du contexte (Guédon, 2009; Lapayre, 2010; Leyrie, 2010).

Ensuite, les mécanismes de structuration et de création de sens des acteurs impliqués sont au centre des préoccupations. Dans cette visée, le projet est perçu comme "un être nomade»; un construit aux frontières floues et perméables, aux moyens et aux fins ambigus ainsi qu'aux résultats escomptés peu spécifiques pour chacun des bénéficiaires (Bonesso, Comacchio et Pizzi, 2014). Le projet est considéré comme une entité au sein de laquelle se développent des pratiques culturelles et des possibilités de changement dans un système souple (Guédon, 2009; Lapayre, 2010; Lemire, Souffez et Laurendeau, 2009).

Dans la perspective contingente et l'agilité systémique, diverses approches de la figure du projet émergent, autant dans les assises ontologiques, épistémologiques, méthodologiques qu'empiriques.

Sur le plan ontologique

Le projet est appréhendé comme une réalité mouvante et adaptative. Cette caractéristique de sujet ondulant procure à la perspective contingente la qualification de meilleures innovations en matière de théorisation en gestion moderne de projet (Guédon, 2009; Plane, 2012; Ruževičius, 2013; Sage et collab., 2013).

\section{Sur le plan épistémologique}

Considérant le projet d'origine pratique et un objet en perpétuel mouvement, les chercheurs ayant inscrit leurs travaux dans la perspective contingente mobilisent, en mode principal, l'avenue constructiviste, postconstructiviste ou pragmatique (Sage et collab., 2014). Ils préconisent quelquefois d'autres perspectives théoriques proches, notamment le socioconstructivisme et l'évolutionnisme, de manière à sortir leurs travaux de recherche des avenues déjà empruntées par leurs prédécesseurs (Gauthier, 2014; Gauthier et Ika, 2012).

\section{Sur les plans théorique et méthodologique}

Les théories des organisations sont régulièrement mobilisées pour soutenir les analyses et les interprétations. Par ailleurs, le devis qualitatif est emprunté en mode principal pour mieux circonscrire la problématique vécue au sein des organisations et ancrée dans de riches contextes, mais complexes. S'inscrivant dans la perspective contingente d'ouverture et de souplesse, les recherches favorisent le dynamisme entre les acteurs et les différents phénomènes de gestion de projet (Guédon, 2009; Hernandez et Belkaid, 2013; Plane, 2012; Richardson, 2015; Ruževičius, 2013).

\section{Sur le plan empirique}

Le champ de gestion de projet s'ouvre aux projets de développement portés par les ONG, par les associations, mais aussi par tous les secteurs de l'économie, de l'industrie et donc de la société, incluant les projets d'art, de sport, de valorisation de la recherche, d'innovation et de capital intellectuel (Asquin, Garel et Picq, 2007; Bredillet et collab., 2010).

La figure 1 synthétise les principales caractéristiques des perspectives universelle et contingente de la gestion moderne de projet. 


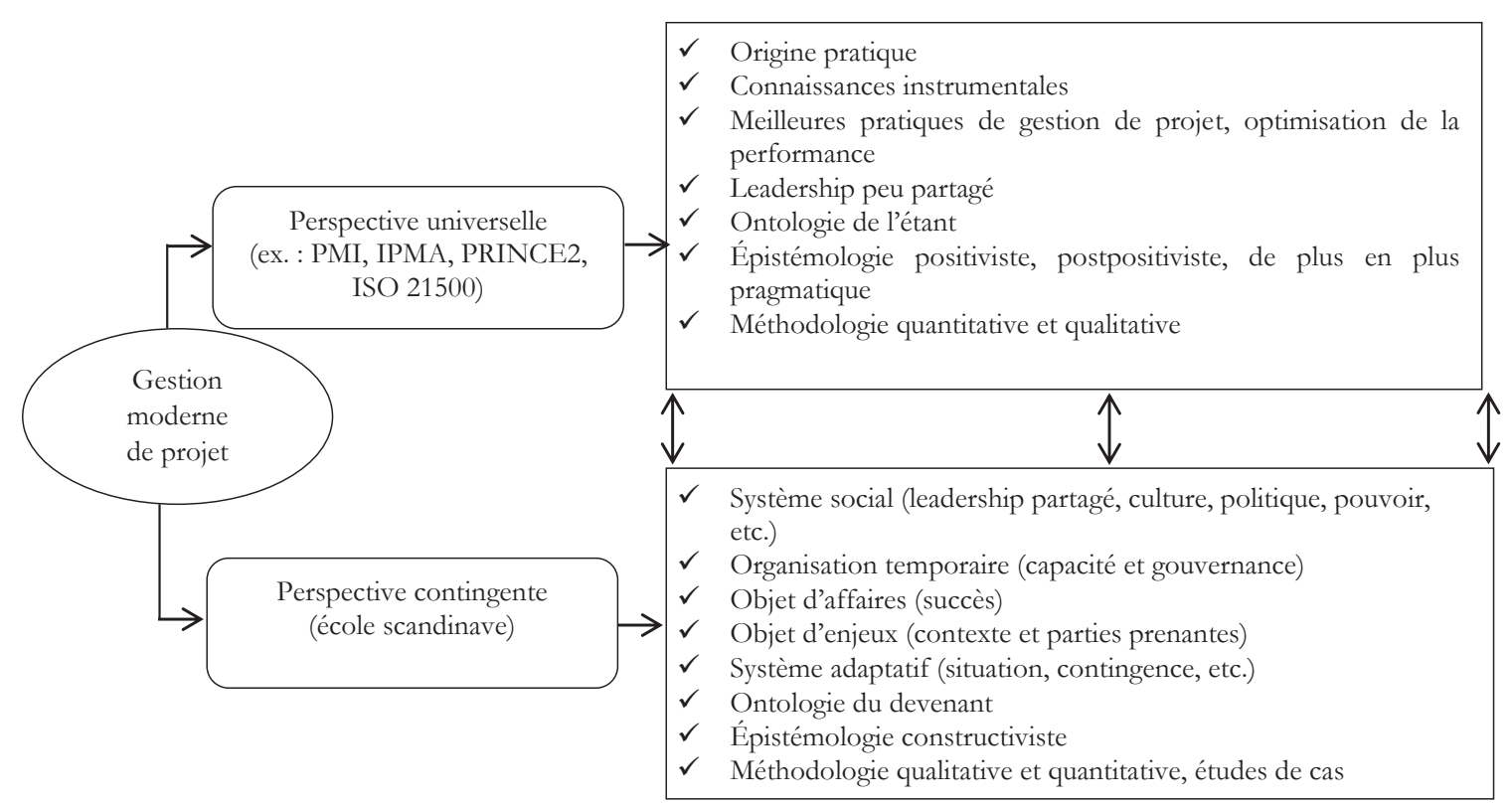

Figure 1 - Principales caractéristiques des deux perspectives de la gestion moderne de projet Source : Compilation des données de la littérature, Mignenan, 2019

Les deux principales avenues théoriques de gestion moderne de projet sont complémentaires. Elles ont pour finalité de répondre à des problématiques spécifiques de manière à générer la performance globale. Les sections suivantes rendent compte des principales compétences généralement exigées chez les acteurs et parties prenantes.

\section{Principales compétences en gestion de projet}

\subsection{Selon la perspective universelle traditionnelle}

Selon plusieurs recherches (Barlatier, 2016; Bredillet et collab., 2010; Kerzner, 2014), les principales facettes de l'approche universelle traditionnelle de la gestion de projet portent pour l'essentiel sur 1) la participation des parties prenantes au processus de gestion du projet, qui est considéré comme cloisonné; 2) la maitrise des dimensions instrumentales du pilotage de projet comme preuve de compétences du gestionnaire, 3) la compréhension des spécificités du contexte du projet et l'adhésion des acteurs à ses objectifs et 4) les compétences sociales et entrepreneuriales des acteurs opérationnels.

En effet, les principaux organismes de normalisation en gestion de projet (IPMA, 2007; PMI, 2008, 2013, 2017) s'accordent sur le fait qu'une des causes majeures des échecs de projets est la faible participation des acteurs au processus cloisonné de gestion. Le fait que les bénéficiaires ne soient pas impliqués dans le cycle de vie du projet entraînerait leur désintéressement remarquable.

Selon le référentiel des organismes de normalisation, l'approche participative dans le processus de projet constitue un des facteurs de réussite (IPMA, 2007; PMI, 2008, 2013, 2017; PRINCE2, 2009). En effet, les professionnels et les théoriciens de la gestion de projet sont unanimes sur le point suivant : la viabilité de tout projet dépend de plus en plus de l'appropriation de son processus par les parties prenantes (Garel, Giard et Midler, 2001). Quoi qu'il en soit, l'acceptabilité et l'appropriation impliquent qu'une forte proportion des parties prenantes collabore aux différentes étapes; que les points de vue de ces dernières soient pris en compte dans le processus de décision. Cela implique l'opérationnalisation d'un dispositif efficace de gouvernance, des pratiques souples de gestion et des avantages à générer (Ahlemann, Hesselmann, Braun et Mohan, 2013; Bekker, 2015; Breese, Jenner, Serra et Thorp, 2015; Eduardo et Serra, 2017; PMI, 2016a, 2016b). Selon quelques auteurs (Blangy et Deffner, 2014), en faisant participer les parties prenantes à un projet, ils s'y sentent acteurs et porteurs du projet. En conséquence, ils accordent plus d'importance à la 
durabilité du résultat escompté et, donc, aux avantages générés. Dans la même perspective, quelques travaux soutiennent que l'intérêt d'adopter l'approche participative porte sur la prise en compte des idées des bénéficiaires (Fernez-Walche et Romon, 2013). Pour ces auteurs, les parties prenantes possèdent des valeurs propres à révéler pour la réussite globale du projet.

La deuxième compétence fréquemment évoquée pour justifier le succès ou l'échec d'un projet porte sur la maitrise des dimensions instrumentales du pilotage (Garel et collab., 2001) par le gestionnaire. Pour les organismes de normalisation, les dimensions instrumentales d'un projet concernent les pratiques de gestion (plans de communication, de trésorerie, chronogramme des activités, gestion des risques, etc.). Elles renvoient à la capacité des acteurs à concevoir et opérationnaliser efficacement les outils de gestion et de pilotage du projet. Les instruments sont consacrés aux visées stratégique et politique et, donc, au contexte du projet (IPMA, 2007; PMI, 2007, 2008, 2013; PRINCE2, 2005, 2009).

De même, la littérature évoque régulièrement la maitrise des champs techniques impliqués dans le projet. Ainsi, la maitrise des principales techniques de gestion de projet fait partie intégrante des compétences des organisations ayant adopté l'approche projet (Garel et collab., 2001). Autrement dit, un gestionnaire de projet doit parvenir à mobiliser ses collaborateurs auprès desquels il a peu de pouvoir hiérarchique et fonctionnel formel.

\subsection{Selon la perspective contingente}

La satisfaction des parties prenantes, la compréhension des spécificités du projet et l'adhésion à ses objectifs (sociaux, d'affaires, etc.) constituent les facettes mises de l'avant par le courant de pensée contingent (Ben Mahmoud Jouini, Charue-Duboc et Midler, 2015; Fernez-Walche et Romon, 2013). En effet, les théories mobilisées par les chercheurs inscrits dans ce courant insistent sur la spécificité du contexte et de la méthodologie à déployer. Le gestionnaire de projet a pour rôle d'éclairer les dimensions contextuelles spécifiques de l'action ainsi que le sens et les priorités qu'il convient de privilégier. Quoi qu'il en soit, selon cette perspective, les compétences culturelles et entrepreneuriales se construisent tout au long du processus. Cependant, cette construction dépend de l'exploration et des compromis des différents aspects ainsi que de la mémoire collective des acteurs.

On est ici bien au-delà d'une démarche d'application d'instrumentation standard puisque la compétence se manifeste dans le réel et dans la pratique (Malai, Brahimi, Farley et Gravel, 2013). De même, la compétence renvoie à la capacité du gestionnaire à sélectionner et adapter les démarches plus efficaces et adaptables à l'écosystème du projet. De ce fait, tout porte à croire que la capacité du gestionnaire (son leadership) à faire saisir le portrait de la vision du projet par les membres de l'équipe est la condition nécessaire pour accroître ses possibilités de succès (Antoine, 2007; Sacramento, 2010).

Enfin, la littérature explorée met en évidence les compétences sociales et entrepreneuriales des acteurs opérationnels. En effet, plusieurs auteurs (Sage et collab., 2014; Sauser et collab., 2009; Turner, Zolin et Remington, 2009) soutiennent que les missions d'un projet se déclinent en responsabilités et en résultats. Cela fait appel à des capacités socioentrepreneuriales des gestionnaires à identifier et formuler les problèmes; à mobiliser les collaborateurs et les méthodes en fonction de leur compréhension et de leur adhésion aux résultats, au contexte et à la vision du projet. Plusieurs recherches recommandent plus spécifiquement les habiletés communicationnelles et le leadership transformationnel pour piloter le projet vers le succès. La diversité des facettes de compétences rend très improbable leur présence chez un seul acteur du projet (Loufrani-Fedida et Missonier, 2015).

Il convient de retenir que, d'une manière générale, les compétences en gestion de projet s'expriment par la participation des parties prenantes, par la maitrise des dimensions instrumentales et par les compétences sociales et entrepreneuriales. La figure 2 rend compte de ces principales compétences. 


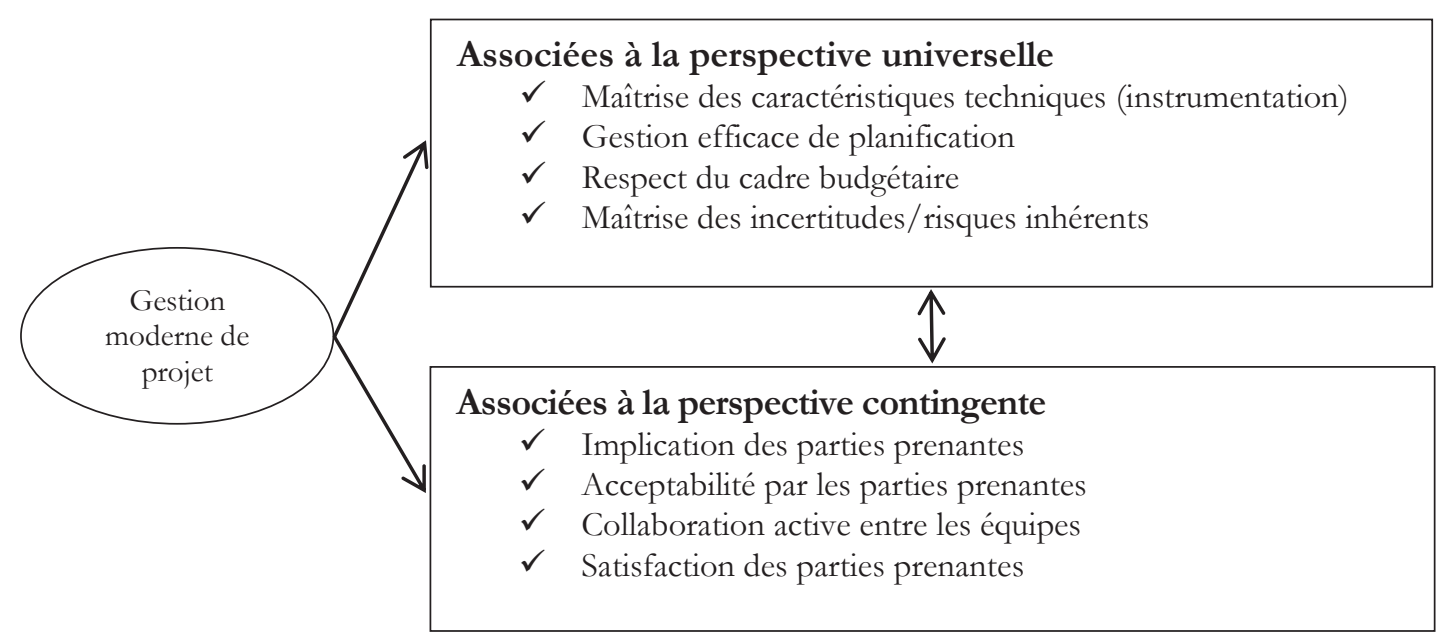

Figure 2-Principales compétences des acteurs selon les deux perspectives de la gestion moderne de projet Source : Compilation des données de la littérature, Mignenan, 2019

\section{Similitudes et divergences des assises ontologiques et épistémologiques selon les perspectives universelle et contingente}

La synthèse de l'approche universelle traditionnelle conceptualisée par Bredillet (2010b) et Söderlund (2004) permet de comprendre que le projet est une entité d'origine pratique et que les compétences du gestionnaire sont mesurées par le niveau de mâ̂trise des connaissances instrumentales. La perspective contingente, théorisée par plusieurs auteurs (Engwall, 2003; Sage et collab., 2013, 2014; Sauser et collab., 2009; Shenhar, 2001), considère aussi le projet comme une pratique humaine dans un contexte bien délimité.

Par contre, les réflexions sur la perspective universelle fondées sur l'ontologie de l'étant et portées par les organismes professionnels de normalisation sont caractérisées par leur univers linéaire. Ces organismes visent l'application rigoureuse et universelle des meilleures pratiques instrumentales pour atteindre une performance élevée de gestion (budget, délai, qualité et risque). Dans cette perspective, les dimensions contextuelles et humaines sont reléguées au second rang dans le processus de modélisation structurelle. Les gestionnaires ont pour rôle le pilotage de planification par le diagramme PERT.
En revanche, la perception qui ressort de la perspective contingente est qu'elle inspire une vision globale pour ainsi saisir le projet dans sa dimension d'entité ondulante "devenant». Les acteurs responsables développent une approche de gestion synchronisée de la qualité et par la qualité (Asquin et collab., 2007; Guédon, 2009; Ruževičius, 2013) pour piloter efficacement le projet. Ce type d'approche est entendu comme une approche systémique dans laquelle des équipes de projet coopèrent activement dans le feu de l'action. De plus, les différences entourant la perspective contingente ont pour visée la recherche permanente du progrès dans la gestion de projet, dans un élan constructiviste ou postconstructiviste et aménagé (Lapayre, 2010). Par ailleurs, c'est une perspective qui, tout en induisant le leadership partagé, la collaboration synchronisée et donc une structure organique et souple, oriente les efforts combinés pour ainsi atteindre la satisfaction élevée des parties prenantes. Enfin, la perspective contingente permet aux acteurs du projet de cheminer ensemble, de cogérer et de co-innover dans la réalité complexe créée par l'écosystème du projet. Elle se préoccupe de la qualité des livrables et des attentes des parties prenantes.

Les éléments de similitude et de divergence présentés à la figure 3 permettent de dégager les fondements théoriques dans lesquels les travaux de recherche en gestion de projet peuvent s'inscrire. 


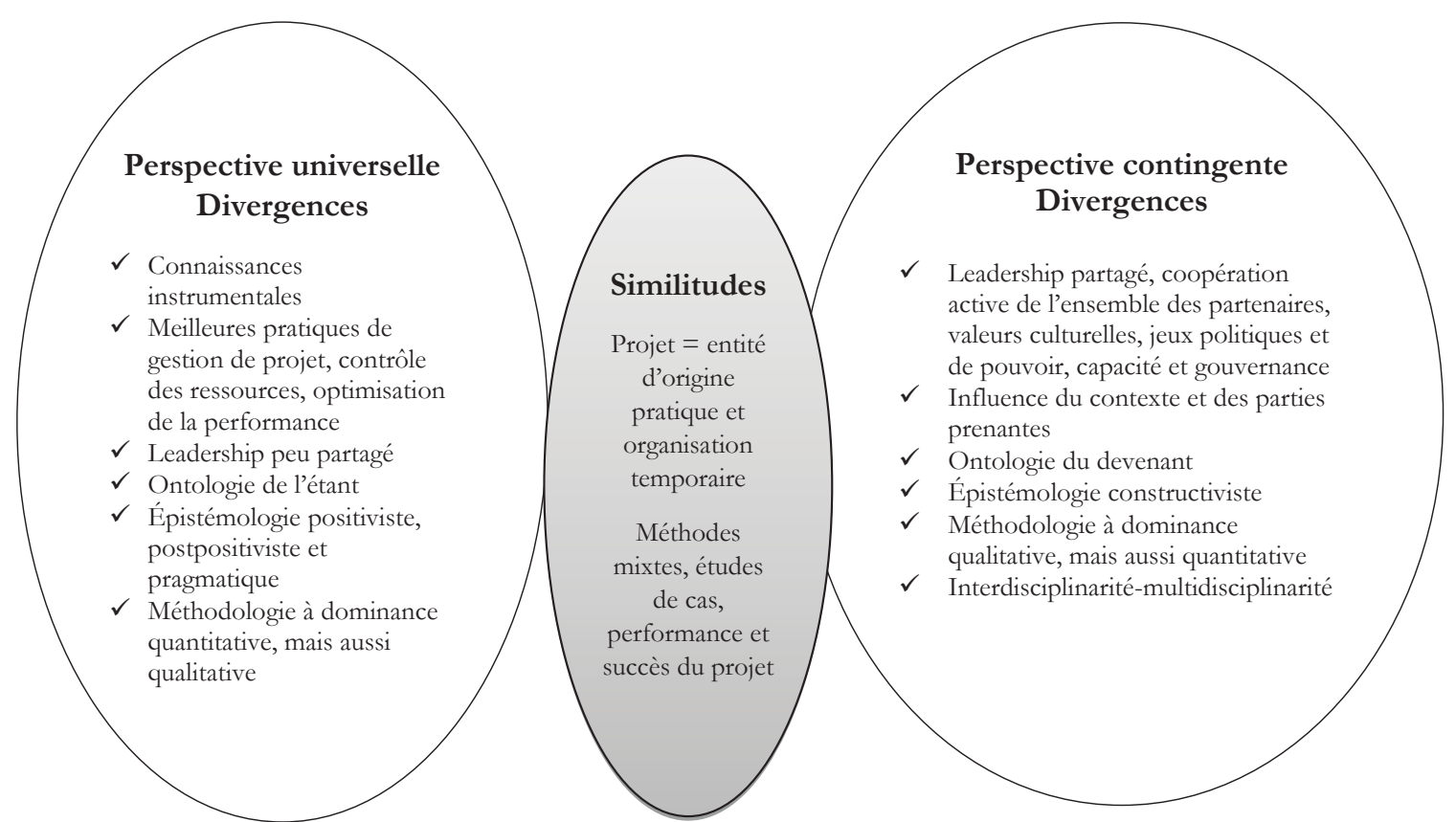

Figure 3 - Éléments de similitude et de divergence des deux perspectives de la gestion moderne de projet Source : Compilation des données de la littérature, Mignenan, 2019

\section{Fondements théoriques au sein desquels les recherches en gestion de projet peuvent s'inscrire}

Quels sont les fondements théoriques en gestion de projet au sein desquels le chercheur peut inscrire son sujet ou qu'il peut adopter? Cette question est importante puisqu'en s'engageant dans une activité d'un mémoire, d'une thèse ou d'un article, le chercheur se lance ainsi en recherche.

En effet, il est admis que les principaux postulats théoriques qui sous-tendent la gestion moderne de projet selon la perspective universelle ou contingente laissent entrevoir, à grands traits, les contours et les lignes de fracture : 1) le courant de pensée universel, à dominance prescriptive, a essentiellement une assise épistémologique positiviste/ postpositiviste et 2) la perspective contingente, de nature descriptive, a un socle épistémologique majoritairement constructiviste/postconstructiviste.

Les postulats prescriptifs de la perspective universelle concernent la planification, la programmation et donc le processus, et sont axés sur le contenu du projet. Ils préconisent l'utilisation des connaissances instrumentales normées et certifiées (p. ex., PMI, IPMA, PRINCE2 et ISO 21500).
La perspective contingente descriptive a un ancrage sur l'écosystème et sur les parties prenantes; elle vise la finalité du projet. Elle s'attache à la coopération active des équipes de projet, à l'importance du capital intellectuel, à l'apprentissage ainsi qu'au développement organisationnel dans l'interaction entre individus et collectivité. De même, elle se préoccupe des relations des parties prenantes en dehors du projet et de la possibilité de leur reproduction à l'intérieur de ce dernier. Elle poursuit l'objectif de contextualisation, d'ancrage spatial et d'encastrement du projet.

En conséquence, le chercheur peut choisir d'adopter, en tenant compte de sa problématique, l'une ou l'autre de ces perspectives. Cependant, ces dernières années, plusieurs recherches empruntent les deux avenues pour traiter la problématique de la performance globale et du succès global de projets.

De tout ce qui précède, il convient de préciser que le projet en tant qu'objet de recherche peut être étudié sous l'angle d'un objet qui « est», qui « devient» ou, à la fois, d'un objet qui « est » et « devient ». Le chercheur, selon sa perspective, pourrait adopter une posture postpositiviste ou postconstructiviste, ou encore un socle épistémique hybride et adopter le "pragmatisme », comme le synthétise le tableau 1. 


\begin{tabular}{|c|c|c|c|}
\hline & $\begin{array}{l}\text { Perspective } \\
\text { universelle }\end{array}$ & $\begin{array}{l}\text { Perspective } \\
\text { contingente }\end{array}$ & $\begin{array}{c}\text { Combinaison des } \\
\text { perspectives }\end{array}$ \\
\hline $\begin{array}{l}\text { Fondement } \\
\text { ontologique }\end{array}$ & $\begin{array}{l}\text { Projet, entité pratique, } \\
\text { une réalité qui existe } \\
\text { (« étant») }\end{array}$ & $\begin{array}{l}\text { Projet, entité pratique, } \\
\text { une réalité qui existe et } \\
\text { évolue (« devenant») }\end{array}$ & $\begin{array}{c}\text { Projet, entité pratique qui « est } \\
\text { et devient» }\end{array}$ \\
\hline $\begin{array}{l}\text { Socle } \\
\text { épistémologique }\end{array}$ & $\begin{array}{c}\text { Positivisme, } \\
\text { postpositivisme }\end{array}$ & $\begin{array}{l}\text { Constructivisme, } \\
\text { postconstructivisme, } \\
\text { évolutionnisme }\end{array}$ & $\begin{array}{c}\text { Postpositivisme, } \\
\text { postconstructivisme et } \\
\text { pragmatisme }\end{array}$ \\
\hline Méthodologies & $\begin{array}{c}\text { Hypothético- } \\
\text { déductive, proposition } \\
\text { de recherche; Études } \\
\text { de cas }\end{array}$ & $\begin{array}{l}\text { Induction, } \\
\text { proposition de } \\
\text { recherche; } \\
\text { Études de cas } \\
\text { longitudinales }\end{array}$ & $\begin{array}{l}\text { Induction-déduction, et vice } \\
\text { versa; Interdisciplinarité; } \\
\text { Multidisciplinarité; } \\
\text { Propositions/hypothèses; } \\
\text { Sondages par enquête, } \\
\text { observations, thèses, études de } \\
\text { cas }\end{array}$ \\
\hline Méthodes & $\begin{array}{l}\text { Sondages par enquête, } \\
\text { observations }\end{array}$ & $\begin{array}{l}\text { Groupes de discussion, } \\
\text { entretiens, observations }\end{array}$ & $\begin{array}{l}\text { Sondages par enquête, } \\
\text { observations, groupes de } \\
\text { discussion, entretiens }\end{array}$ \\
\hline $\begin{array}{l}\text { Terrain de } \\
\text { manœuvre et } \\
\text { sources de } \\
\text { données }\end{array}$ & \multicolumn{3}{|c|}{$\begin{array}{l}\text { Projets de développement collectif; d'entreprise; d'énergie; d'informatique (TIC); } \\
\text { miniers; culturels; de construction d'usine; d'entreprises de génie-conseil; de transfert } \\
\text { technologique; d'innovation, etc. }\end{array}$} \\
\hline
\end{tabular}

Tableau 1 - Synthèse des approches de gestion de projet Source : Compilation des données de la littérature, Mignenan, 2019

\section{Conclusion}

Le présent article avait pour objectifs de dresser le portrait du fondement théorique de deux perspectives en gestion moderne de projet et de présenter les assises sur lesquelles les recherches dans ce champ pourraient reposer.

Il en ressort que la perspective universelle, tout en considérant le projet comme un objet pratique, formalise le savoir-faire et les règles de l'art en matière de gestion de projet. À travers son corpus de connaissances, elle documente les meilleures pratiques et les pratiques fondamentales de la gestion de projet. La perspective contingente, quant à elle, préconise la gestion collaborative, la synergie entre l'ensemble des acteurs et l'autonomie des équipes de projet pour aboutir à la satisfaction des parties prenantes.

Sans inscrire les recherches en gestion de projet dans l'une ou l'autre de ces perspectives, il convient de préciser tout simplement que la recherche dans ce champ au sein des organisations s'inscrit dans l'ensemble des activités de management de projet (MP). De ce fait, en examinant les relations de complémentarité entre l'assise théorique de la perspective universelle (Gauthier et Ika, 2012) et contingente (Sauser et collab., 2009; Shenhar, 2001) de la gestion de projet, tout chercheur est en mesure d'identifier clairement l'enseigne dans laquelle il inscrira ses travaux de recherche.

Toutefois, l'instabilité et l'hétérogénéité des paramètres théoriques et historiques des fondements de la gestion de projet de même que l'incapacité d'en venir à un consensus nous empêchent d'en arriver à des conclusions systématiques. Cependant, nous croyons qu'il est possible, même dans ce bref exposé, que les chercheurs trouvent des éléments leur servant de canevas plus fructueux pour leur permettre de s'approprier leur recherche. Cela dit, ce travail n'a pas pour but de tracer la trajectoire théorique aux chercheurs en gestion de projet, mais peut servir de guide pour structurer les réflexions sur le cadre théorique en gestion de projet.

\section{NOTES}

1 L'agilité est un mode de gestion de projet qui met de l'avant la collaboration synchronisée des individus, l'amélioration continue, l'innovation constante et la remise en question pour répondre véritablement aux besoins du client : se concentrer davantage sur l'adaptation au changement que sur le suivi et le respect d'un plan de recherche de la valeur ajoutée. 
2 L'école de pensée de Francfort, portée par les sciences sociales, est caractérisée par sa négation irréconciliable avec l'ordre établi, son antipositivisme passionné, son absence de neutralité axiologique, son caractère partisan, son adhésion à des présuppositions, sa posture relativiste, etc. (Löwy, 1982).

\section{RÉFÉRENCES}

Ahlemann, F., Hesselmann, F., Braun, J. et Mohan, K. (2013). Exploiting is/it projects' potential: Towards a design theory for benefits management. ECIS 2013 Completed Research, 210. Repéré à http://aisel.aisnet.org/ecis2013_cr/210

Antoine, M. (2007). Le succès des projets d'innovation inter-organisationnels initiés dans le cadre d'une politique publique. Entrepreneuriat et innovation, 11(2), 61.

Asquin, A., Falcoz, C. et Picq, T. (2005). Ce que manager par projet veut dire : comprendre, comment faire, prendre du recul. Paris, France : Éditions d'Organisation.

Asquin, A., Garel, G. et Picq, T. (2007). Quand les individus et les collectifs sociaux sont mis en danger par le travail en projet. Gérer et comprendre, 43-54. Repéré à https:// halshs.archives-ouvertes.fr/halshs-00687924/document

Asquin, A., Garel, G. et Picq, T. (2009, mai). When project-based management causes distress at work. Communication présentée à la 9e Conférence de l'EURAM, Liverpool, Royaume-Uni. Repéré à https:// halshs.archives-ouvertes.fr/halshs-00688866/document

Asquin, A., Garel, G. et Picq, T. (2010). When project-based management causes distress at work. International Journal of Project Management, 28(2), 166-172. https://doi.org/10.1016/j.ijproman.2009.08.006

Badewi, A. et Shehab, E. (2015). The impact of organizational project benefits management governance on ERP project success: Neo-institutional theory perspective. International Journal of Project Management, 34(3), 412-428. https://doi.org/10.1016/ j.ijproman.2015.12.002

Barlatier, P.-J. (2016). Management de l’innovation et nouvelle ère numérique : enjeux et perspectives. Revue française de gestion, 254(1), 55-63. https://doi.org/10.3166/rfg.2016.00009

Bekker, M. (2015). Project governance: The definition and leadership dilemma. Procedia - Social and Behavioral Sciences, 194, 33-43. https://doi.org/10.106/j.sbspro.2015.06.117

Ben Mahmoud Jouini, S., Charue-Duboc, F. et Midler, C. (2015). Management de Pinnovation et globalisation : enjeux et pratiques contemporains. Paris, France : Dunod.

Blangy, S. et Deffner, A. (2014). Impacts du développement minier sur les hommes et les caribous à Qamani'tuaq au Nunavut : approche participative. Études Inuit, 38(1-2), 239-265. https://doi.org/10.7202/1028862ar

Bonesso, S., Comacchio, A. et Pizzi, C. (2014). Project-based knowledge in organizing open innovation. Londres, R.-U. : Springer.

Boutinet, J.-P. (2005). Anthropologie du projet. Paris, France : Presses universitaires de France.

Bredillet, C. N. (2006). The future of project management: Mapping the dynamics of project management field in action. Dans D. L. Cleland et R. Gareis (dir.), Global project management handbook: Planning, organiring, and controling international project (p. 65-89). New York, NY : McGraw-Hill Professional.

Bredillet, C. N., Yatim, F. et Ruiz, P. (2010). Project management deployment: The role of cultural factors. International Journal of Project Management, 28, 183-193. https://doi.org/10.1016/j.ijproman.2009.10.007

Bredillet, C.N. (2010b). Mapping the Dynamics of the Project Management Field: Project Management in Action (Part 6). Project Management Journal, 41( 2), 2-4

Breese, R., Jenner, S., Serra, C. E. M. et Thorp, J. (2015). Benefits management: Lost or found in translation. International Journal of Project Management, 33(7), 1438-1451. https://doi.org/10.1016/j.ijproman.2015.06.004

Camilleri, E. (2011). Project success: Critical factors and behaviours. Aldershot, R.-U. : Gower.

Chia, R. (1995). From modern to postmodern organisational analysis. Organisational Studies, 16(4), 579-604. https://doi.org/10.1177/017084069501600406

Chih, Y.-Y. et Zwikael, O. (2015). Project benefit management: A conceptual framework of target benefit formulation. International Journal of Project Management, 33(2), 352-362. https://doi.org/10.1016/j.ijproman.2014.06.002 
Cicmil, S. (2006). Understanding project management practice through interpretative and critical research perspectives. Project Management Journal, 37(2), 27-37. https://doi.org/10.1177/875697280603700204

Cicmil, S. et Hodgson, D. (2006). New possibilities for project management theory : A critical engagement. Project Management Journal, 37(3), 111-122. https://doi.org/10.1177/875697280603700311

Eduardo, C. et Serra, M. (2017). Benefits realization management: Strategic value from portfolios, programs, and projects. Boca Raton, FL : Taylor \& Francis Group.

Engwall, M. (2003). No project is an island: Linking projects to history and context. Research Policy, 32(5), 789-808. https://doi.org/10.1016/S0048-7333(02)00088-4

Fernez-Walche, S. et Romon, F. (2013). Management de Pinnovation : de la stratégie aux projets. Paris, France : Vuibert.

Festel, G. (2013). Technology transfer models between industrial biotechnology companies and academic spin-offs. Journal of Industrial Biotechnology, 9(5). http://doi.org/10.1089/ind.2013.1590

Garel, G. (2011a). Le management de projet. Paris, France : La Découverte.

Garel, G. (2011b). Qu'est-ce que le management de projet? Informations sociales, 167(5), 72-80. Repéré_à www.cairn.info/revue-informations-sociales-2011-5-page-72.htm

Garel, G. (2013). A history of project management models: From pre-models to the standard models. International Journal of Project Management, 31(5), 663-669. https://doi.org/10.1016/j.ijproman.2012.12.011

Garel, G., Giard, V. et Midler, C. (2001). Management de projet et gestion des ressources humaines. Paris, France : GREGOR. Repéré à www.lamsade.dauphine.fr/ giard/2001-05.pdf

Gauthier, J.-B. (2014). Fondements de la recherche en gestion de projet : perspective réflexive pour un nowvel éclairage. Québec, QC : Université du Québec en Outaouais. Repéré à http://di.uqo.ca/id/eprint/777

Gauthier, J.-B. et Ika, L. A. (2012). Foundations of project management research : An explicit and six-facet ontological framework. Project Management Journal, 43(5), 5-23. https://doi.org/10.1002/pmj.21288

Guédon, J. (2009). Qu'est-ce qu'une approche critique en management de projet? Une perspective ontologique. Cabier de recherche/LAE Normandie, 64, 1-23.

Hernandez, S. et Belkaid, E. (2013). L'influence du contexte sur le management territorial en Méditerranée : une analyse comparée de Barcelone, Marseille et Tlemcen. Management et Avenir, 5(63), 145-164. https://doi.org/10.3917/mav.063.0145

IPMA. (2007). Project perspectives 2009 : The Annual Publication of International Project Management Association (vol. XXXI), Lisbonne, Portugal : IPMA.

Kerzner, H. (2014). Project management best practices: Achieving global excellence. Hoboken, NJ : John Wiley \& Sons.

Lapayre, N. (2010). Une analyse critique du management de projet : quels enseignements pour la gestion des ressources bumaines? Communication présentée au 21 ${ }^{\mathrm{e}}$ Congrès de l'AGRH, Rennes/Saint-Malo, France.

Lemire, N., Souffez, K. et Laurendeau, M.-C. (2009). Animer un processus de transfert des connaissances : bilan des connaissances et outil d'animation. Québec, QC : Institut national de la santé publique. Repéré à www.inspq.qc.ca/pdf/publications/ 1012_AnimerTransfertConn_Bilan.pdf

Leyrie, C. (2010). La politique dans les projets comme compétence collective : proposition d'un modèle multi niveau et multidimensionnel. Communication présentée au 21 ${ }^{\mathrm{e}}$ Congrès de l'AGRH, Rennes/Saint-Malo, France.

Loufrani-Fedida, S. (2011). La gestion des ressources humaines au service de l'articulation entre management des compétences et organisation par projets. Revue de gestion des ressources bumaines, 76(1), 24-38. https://doi.org/10.3917/grhu.079.0024.

Loufrani-Fedida, S. et Missonier, S. (2015). The project manager cannot be a hero anymore! Understanding critical competencies in project-based organizations from a multilevel approach. International Journal of Project Management, 33(6), 1220-1235. https://doi.org/10.1016/j.ijproman.2015.02.010

Löwy, M. (1982). Le marxisme rationaliste de l'École de Francfort. L'Homme et la société, 65-66, 45-65. Repéré à www.persee.fr/doc/homso_0018-4306_1982_num_65_1_2089 
Malai, D., Brahimi, C., Farley, C. et Gravel, F. (2013). Competency framework. for scientific processes underlying the cross-cutting functions of the Institut national de santé publique du Québec. Montréal, QC : Institut national de santé publique. Repéré à www.inspq.qc.ca/node/3751

Mignenan, V. (2019). Modèle de management de projets de transfert technologique et d'innovation, thèse de doctorat en management de projets, UQAC, proquest.com. 345 p.

Morris, P., Pinto, J. et Söderlund, J. (2011). The Oxford handbook of project management. Oxford/ Toronto : Oxford University Press.

Morrissette, J. (2013). Recherche-action et recherche collaborative : quel rapport aux savoirs et à la production de savoirs? Nowvelles pratiques sociales, 25(2), 35-49. https://doi.org/10.7202/1020820ar

Müller, J.-L. G. (2005). Les fondamentaux du management de projet : 100 questions pour comprendre et agir. Paris, France : AFNOR.

Padgett, C. (2009). The project success method: A proven approach for achieving superior project performance in as little as 5 days. Hoboken, NJ : John Wiley \& Sons.

Plane, J.-M. (2012). Théorie et management des organisations. Paris, France : Dunod.

PMI. (2007). Practice standard for project configuration management. Newtown Square, PA: PMI.

PMI. (2008). Guide du corpus des connaissances en management de projet (4e éd.). Newtown Square, PA: PMI.

PMI. (2013). A guide to the project management body of knowledge (5e éd.). Newtown Square, PA: PMI.

PMI. (2016a). Delivering value: Focus on benefits during project execution. Newtown Square, PA: PMI.

PMI. (2016b). Governance of portfolios, programs, and projects: A practice guide. Newtown Square, PA: PMI.

Repéré à https://fr.scribd.com/book/343645877/Governance-of-Portfolios-Programs-and-Projects-A-Practice-Guide

PMI. (2017). Guide du corpus des connaissances en management de projet (6e éd.). Newtown Square, PA : PMI.

PRINCE2. (2005). Managing success projects with PRINCE2. Londres, R.-U. : OGC.

PRINCE2. (2009). Managing successful projects with PRINCE2. Londres, R.-U. : OGC.

Repéré à www.academia.edu/7608654/Managing_Successful_Projects_with_PRINCE2_2009

Richardson, G. L. (2015). Project management : Theory and practice. New York, NY : Taylor \& Francis Group.

Ruževičius, J. (2013). Changements de paradigme du management de la qualité. International Business : Innovations, Psychology, Economics, 4(6), 33-44. Repéré à http://www.kv.ef.vu.lt/wp-content/uploads/2010/10/STRAIPSNIS-Changements-de-paradigme-dumanagement-de-la-qualite.pdf

Sacramento, D. (2010). Le succès des projets de développement international: analyse de la qualité de la relation entre le chef de projet de Pentreprise sous-traitante et le coordonnateur national du projet. Montréal, QC : Université du Québec à Montréal. Repéré à https://archipel.uqam.ca/3740/1/M11655.pdf

Sage, D., Dainty, A. et Brookes, N. (2013). Thinking the ontological politics of managerial and critical performativities: An examination of project failure. Scandinavian Journal of Management, 29(3), 282-291. https://doi.org/10.1016/ j.scaman.2013.01.004

Sage, D., Dainty, A. et Brookes, N. (2014). A critical argument in favor of theoretical pluralism: Project failure and the many and varied limitations of project management. International Journal of Project Management, 32, 544-555. https://doi.org/10.1016/j.ijproman.2013.08.005

Sauser, B., Reilly, R. et Shenhar, A. (2009). Why projects fail? How contingency theory can provide new insights: A comparative analysis of NASA's Mars Climate Orbiter loss. International Journal of Project Management, 27, 665-679. https://doi.org/10.1016/j.ijproman.2009.01.004

Shenhar, A. J. (2001). One size does not fit all projects Exploring classical contingency domains. Management Science, 47(3), $394-414$. https://doi,org/10.1287/mnsc.47.3.394.9772

Slevin, D. (2002). The frontiers of project management research. Newtown Square, PA: PMI.

Söderlund, J. (2004). Building theories of project management: Past research, questions for the future. International Journal of Project Management, 22(3), 183-191. https://doi.org/10.106/S0263-7863(03)00070-X 
Söderlund, J. (2011). Pluralism in project management: Navigating the crossroads of specialization and fragmentation. International Journal of Management Reviens, 13(2), 153-176. https://doi.org/10.1111/j.1468-2370.2010.00290.x

Söderlund, J. et Geraldi, J. (2012). Classics in project management: Revisiting the past, creating the future. International Journal of Managing, 5(4), 559-577. https://doi.org/10.1108/17538371211280245

Špundaka, M. (2014). Mixed agile/traditional project management methodology: Reality or illusion? Procedia - Social and Behavioral Sciences, 119, 939-948. https://doi.org/10.1016/j.sbspro.2014.03.105

Too, E. G. et Weaver, P. (2014). The management of project management: A conceptual framework for project governance. International Journal of Project Management, 32(8), 1382-1394. https://doi.org/10.1016/j.ijproman.2013.07.006

Turner, R. (2010). Perspectives on projects. Londres, R.-U.: Routledge.

Turner, R. (2014). Gower handbook of project management. Farnham, R.-U. : Gower.

Turner, R., Huemann, M. et Keegan, A. (2008). Human resource management in the project-oriented organization. Newtown Square, PA: PMI.

Turner, R., Zolin, R. et Remington, K. (2009, octobre). Monitoring the performance of complex projects from multiple perspectives over multiple time frames. Communication présentée à la 9e Conférence de l'IRNOP, Berlin, Allemagne. Repéré à https://eprints.qut.edu.au/29010/ 